Portland State University

PDXScholar

Electrical and Computer Engineering Faculty

Publications and Presentations

$5-4-2011$

\title{
Terahertz Material Detection From Diffuse Surface Scattering
}

\author{
Scott Schecklman \\ Portland State University \\ Lisa M. Zurk \\ Portland State University, zurkl@pdx.edu \\ Samuel C. Henry \\ Portland State University \\ Gabriel Paul Kniffin \\ Portland State University
}

Follow this and additional works at: https://pdxscholar.library.pdx.edu/ece_fac

Part of the Electrical and Computer Engineering Commons

Let us know how access to this document benefits you.

\section{Citation Details}

Schecklman, S., Zurk, L. M., Henry, S., \& Kniffin, G. P. (2011). Terahertz material detection from diffuse surface scattering. Journal of Applied Physics,109(9), 094902-094902.

This Article is brought to you for free and open access. It has been accepted for inclusion in Electrical and Computer Engineering Faculty Publications and Presentations by an authorized administrator of PDXScholar. Please contact us if we can make this document more accessible: pdxscholar@pdx.edu. 


\title{
Terahertz material detection from diffuse surface scattering
}

\author{
Scott Schecklman, ${ }^{a}$ Lisa M. Zurk, Samuel Henry, and Gabriel P. Kniffin \\ Department of Electrical and Computer Engineering, Portland State University, Portland, Oregon 97207, USA
}

(Received 11 October 2010; accepted 16 November 2010; published online 4 May 2011)

\begin{abstract}
The potential for terahertz ( $\mathrm{THz}$ ) spectroscopy to detect explosives and other materials of interest is complicated by rough surface scattering. Our previous work has demonstrated that by averaging over diffuse observation angles and surfaces, spectral features could be recovered from laboratory measurements and numerical computer simulations. In addition to averaging, a low-pass cepstrum filter was used to reduce noise due to the random rough surface. This paper expands on these concepts by using the cepstrum of both the random rough surface and the material properties of the target material to choose an optimal cutoff frequency for the filter. The utility of these techniques is evaluated using laboratory measurements and Monte Carlo simulations for many sets of random surface realizations. The Kirchhoff Approximation is used to quickly model diffuse scattering from dielectric materials with gradually undulating rough surfaces when the incident and diffuse scattering angles are near the surface normal. The ability to recover the spectral features of rough dielectric materials from diffuse $\mathrm{THz}$ scattering may prove useful for the design of future security screening systems. (C) 2011 American Institute of Physics. [doi:10.1063/1.3561806]
\end{abstract}

\section{INTRODUCTION}

During the 20th century many technological advances were made using electromagnetic waves in both the microwave and optical regions of the spectrum. However, the band of frequencies between these two regions, often referred to as the terahertz $(\mathrm{THz})$ gap, ${ }^{1}$ remained difficult to access due to the technical challenges of generating and detecting waves at the far edges of the microwave and optical portions of the spectrum. Recent advances in laser technology have overcome many of these hurdles and pulsed $\mathrm{THz}$ systems have now achieved bandwidths spanning five octaves (0.1-3.0 THz). These advancements have opened up a new avenue to explore the material properties in the farinfrared portion of the electromagnetic spectrum.

Materials composed of polar molecules are of particular interest since they possess characteristic resonant frequencies which alter the frequency-dependent refractive index of the material in a dramatic but predictable manner; tracing out unique spectroscopic signatures in the material's absorption spectra, which can be used for material identification. ${ }^{2}$ In contrast, nonpolar molecules have no such resonances in the $\mathrm{THz}$ band and are mostly transparent within the $\mathrm{THz}$ spectrum. Terahertz waves are also nonionizing and are considered safe for human exposure at low power levels. ${ }^{1,3}$

These properties suggest a number of promising applications for $\mathrm{THz}$ technology. Of particular interest is the development of security screening systems to detect concealed explosives and illicit drugs. Terahertz waves have the unique ability to travel through (nonpolar) clothing and packaging materials, interact with (polar) materials of interest, and then return to a detector with a characteristic spectral signature. This signature could be used to identify the contents of packages as they make their way through postal systems and airports.

${ }^{\text {a)} E l e c t r o n i c ~ m a i l: ~ s s c h e c k @ p d x . e d u . ~}$
While laboratory experiments have revealed the potential benefits of $\mathrm{THz}$ technology, two important challenges must be considered before practical detection systems can be employed in realistic screening systems. First, most materials of interest are opaque at $\mathrm{THz}$ frequencies, and it is therefore expected that detection systems must operate in a reflection configuration. ${ }^{4}$ Second, many explosives contain granular inhomogeneities on the order of hundreds of microns in diameter. ${ }^{5-7}$ Such particles are comparable to $\mathrm{THz}$ wavelengths and could therefore cause frequency-dependent volume scattering, as well as scattering from the material's rough surface - modulating the spectral signature and complicating material identification.

For a given transmitter and receiver configuration, the strength of a wave reflected from a rough surface will depend on the material properties (the refractive index for nonmagnetic materials) and "noise" introduced by the random rough surface. A hypothetical $\mathrm{THz}$ screening system is illustrated in Fig. 1, where a single $\mathrm{THz}$ transmitter (Tx) illuminates a sample, and a number of $\mathrm{THz}$ receivers $(\mathrm{Rx})$ above the rough surface collect the diffuse scattered power from a number of independent viewpoints. If the sample material is allowed to move laterally beneath the scanner (such as on a conveyor belt for luggage or mailed packages), then many independent sample areas on the random rough surface could be illuminated. If materials of interest are homogeneous and anisotropic, then the signature in the material parameters can be recovered by averaging many measurements from uncorrelated spots on the surface and/ or different, uncorrelated diffuse angles. Before averaging the received signals, a phase shift would typically be applied to each of the individual receivers to account for differences in their respective propagation paths. To simplify the discussion, the illustration in Fig. 1 shows the receiver elements configured in an arc so that the path between the illuminated surface and each receiver is the same. 


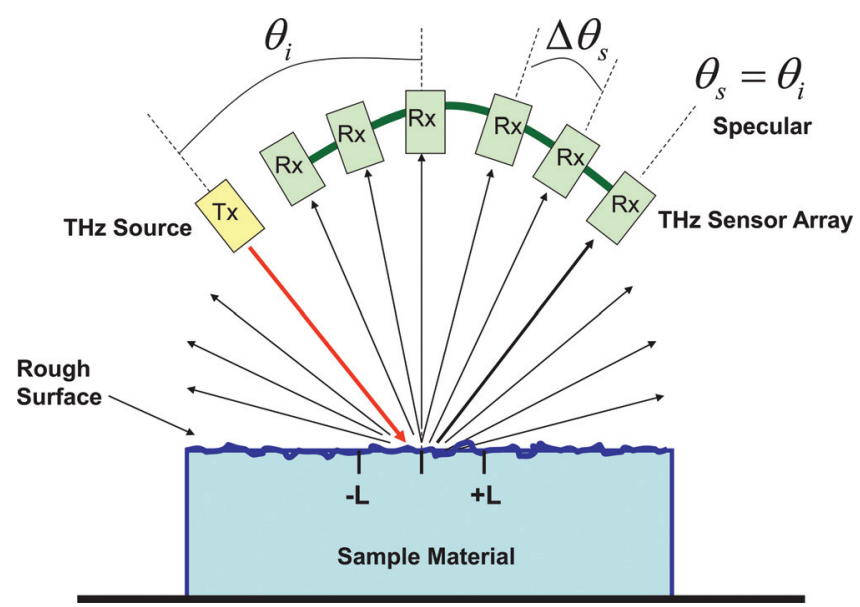

FIG. 1. (Color online) A hypothetical THz TDS system configured to measure diffuse scattering from a material with a random rough surface. The sample moves laterally beneath the detector array which takes snapshots of independent illuminated surfaces of length, $2 L$. Averaging over the uncorrelated receiver angles and surface snapshots reduces the noise introduced by the random rough surface and enhances the correlated material properties.

Such averaging techniques have been applied experimentally for lactose impressed with a rough surface ${ }^{8,9}$ and in Finite Difference Time Domain (FDTD) scattering simulations. ${ }^{10}$ The latter work also introduced the concept of applying a low-pass filter to the Fourier Transform of the scattered power as a smoothing technique to reduce the scattering noise and improve the visibility of $\mathrm{THz}$ spectral features. The performance a Gaussian filter was evaluated using various standard deviations set to a fraction of the total bandwidth.

The work presented here expands on these concepts by considering a method to choose a standard deviation for the Gaussian low-pass filter. The Fourier Transform of a frequency spectrum is often called a "cepstrum", where the first four letters of the word, "spectrum" have been reversed. Similarly, the domain of the cepstrum is sometimes referred to as the "quefrency" domain by rearranging the first six letters of the word "frequency". ${ }^{11}$ This terminology will be used throughout the remainder of this paper. Thus, we seek a method to locate an optimal cutoff quefrency for a Gaussian low-pass cepstrum filter. It will be shown that information about the extinction coefficient and surface roughness of a target material may be used to reduce noise introduced by rough surface scattering and improve detection of the material of interest.

In this paper the effects of averaging and cepstrum filtering are evaluated in Monte Carlo simulations for many sets of random rough surface realizations. The following section briefly reviews the laboratory techniques used to obtain spectroscopic information from dielectric materials with random rough surfaces. The Kirchhoff Approximation is used to model diffuse scattering at angles near the surface normal for random rough dielectric and conductive surfaces. Section III describes the procedures used to create rough surface samples and measure the $\mathrm{THz}$ scattering at diffuse angles. Averaging and cepstrum filtering concepts are discussed in Sec. IV. In Sec. V, detection methods are evaluated for laboratory measurements and computer simulations.

\section{ROUGH SURFACE SPECTROSCOPY MODEL}

Explosives are one of the primary materials of interest for $\mathrm{THz}$ spectroscopy. These materials may be composed of granular materials with particles on the order of hundreds of microns. ${ }^{5}$ Thus, the surfaces of these materials may have roughness on the same order as $\mathrm{THz}$ wavelengths, resulting in rough surface scattering. ${ }^{12-14}$ In this section, rough surface scattering concepts and terminology are discussed and a method for recovering spectral signatures using $\mathrm{THz}$ scattering from materials with rough surfaces is considered.

\section{A. THz reflection spectroscopy}

Terahertz Time Domain Spectroscopy (TDS) systems use emitters which produce a brief current pulse which is then radiated by broadband antenna and focused onto a sample for either a transmission or reflection measurement. A similar lens and antenna collect the transmitted or reflected waves in a coherent detection scheme. The Fourier transform of the narrow time pulse covers a wide bandwidth extending up to $\mathrm{THz}$ frequencies.

In THz-TDS systems, it is not possible to accurately account for the transfer functions of all of the individual emitter and detector components. Normalizing the sample measurement by a reference measurement in the frequency domain removes these unknowns and isolates the material parameters in a process called deconvolution. In a transmission configuration, the reference measurement is simply done without the sample in place. ${ }^{15}$ Laboratory transmission measurements of thin samples have been used to catalog spectroscopic signatures of many materials. ${ }^{2,16-18}$

In reflection spectroscopy, the reference measurement is performed by replacing the sample with a higly reflective mirror. ${ }^{15}$ However, it is not practical to expect that the mirror will always be placed at the exact same location as the sample (to within a fraction of a $\mathrm{THz}$ wavelength), and a phase difference between the sample and mirror measurements will introduce some error into the extracted material properties. ${ }^{15}$ While it is possible to correct for the phase alignment in carefully controlled laboratory measurements, it is unlikely that accurate phase information can be obtained in a practical THz security screening system.

However, for many materials of interest, the reflected intensity can provide a spectroscopic signature which is quite similar to that of the absorption without need of the reflected phase. For solid, polar dielectric materials that are made up of molecules with vibrational or rotational oscillations, the Lorentz model may be used to describe the resonance in the refractive index. Many explosive related compounds (ERC's) fit this description and also have strong dispersion relative to absorption. ${ }^{19,20}$ For such materials the negative derivative of the magnitude of the reflection coefficient reveals spectral peaks at frequencies near the peaks in the extinction coefficient. ${ }^{19,20}$ Although the relative height of the peaks may be altered, the frequencies of the peaks in the derivatives are near the peak frequencies in the extinction coefficient. ${ }^{19,20}$ Since the reflectivity of such materials is simply the squared magnitude of the reflection coefficient, it is possible to qualitatively compare the derivative of the 
reflectivity with the absorption signatures obtained from a database of transmission measurements and identify a target material. This method has been useful on flat surfaces at normal incidence. ${ }^{19-21}$ It has also been applied experimentally to lactose impressed with a rough surface ${ }^{8,9}$ and to scattering simulations with $\mathrm{C} 4$ explosive. ${ }^{10}$

It is important to note that when using the derivative of the reflected intensity (as described above), a deconvolution of the sample measurement with a reference mirror is still necessary to cancel the transfer functions of the $\mathrm{THz}$ transmitter and receiver components. After the deconvolution, the derivative of the intensity may be used to identify materials without accounting for the phase difference between the sample and reference paths. The next section will briefly introduce rough surface scattering concepts and a method for simulating rough surface scattering from dielectric surfaces.

\section{B. Surface scattering model}

The previous discussion explained how the derivative of the reflectivity could reveal spectral features for some ERC's with flat surfaces. This section briefly discusses random rough surface scattering concepts, and introduces a method for modeling diffuse scattering from a dielectric material in the configuration illustrated in Fig. 1. This discussion assumes that the surface is level and that the material is thick enough so that it attenuates any internal reflections and effectively acts as an infinite half-space. In addition, the sample material is assumed to be uncovered and in an atmosphere with no absorption.

The impact of the surface roughness on the scattering depends on the surface height relative to a wavelength. Thus, as wavelength decreases (with increasing frequency), a surface will appear more rough to the incident wave and result in more diffuse scattering. The Fraunhofer Criterion is often used to determine the rms surface height that will cause significant scattering. ${ }^{22,23}$ Thus, a surface will cause rough surface scattering if its rms height, $h$, satisfies the inequality,

$$
h \geq \frac{\lambda}{32 \cos \theta_{i}},
$$

where $\theta_{i}$ is the angle of incidence relative to the surface normal and $\lambda$ is the wavelength.

Huygens' Principle may be used to sum the scattered fields from all the points along a rough surface to give the total scattered field, $E^{s}$, at a point far above the rough surface. The Kirchhoff Approximation (KA) or Tangent Plane Approximation then treats the facets of the rough surface as tangent planes and applies the Fresnel reflection coefficient to calculate the local scattered fields. In doing so, this model neglects shadowing by the peaks of the surface as well as multiple scattering within the valleys. Therefore, the KA model is often applied to surfaces that are gradually undulating relative to the incident wavelength, and in scenarios where the incident and scattered angles are near the surface normal. A complete derivation of the Kirchhoff Approximation for a one-dimensional dielectric surfaces can be found in the literature. ${ }^{22,24-26}$
Under the previous assumptions, the total scattered field from a one-dimensional rough surface of height, $\zeta(x)$ extending from $-L$ to $L$ is given by, ${ }^{22}$

$$
\tilde{E}^{s \pm}=\frac{\mp 1}{4 L \cos \theta_{i}} \int_{-L}^{L}\left(a \zeta_{x}^{\prime}-b\right) e^{i v_{x} x+i v_{z} \zeta} d x,
$$

where $E^{s}$ is the scattered field from a rough surface, normalized by the reflected field from a perfectly smooth conducting surface, ${ }^{22}$ and $(+,-)$ represent vertical and horizontal polarization, respectively. Throughout this paper the tilde is used to indicate a complex quantity. In Eq. $2, \zeta_{x}^{\prime}$ is the derivative of the surface profile with respect to position $x$. The phase shift due to surface roughness, $\zeta(x)$, and the transmitter and receiver configuration, $\theta_{i}$ and $\theta_{s}$, is dictated by the exponential terms, where $v_{x}=k\left(\sin \theta_{i}-\sin \theta_{s}\right)$ and $v_{z}=-k\left(\cos \theta_{i}+\cos \theta_{s}\right)$. In this paper we assume the material above the surface is dry air (no absorption) so that the wavenumber, $k=2 \pi / \lambda$ is a real quantity. The material properties of the surface are accounted for within the coefficients $a=(1-\tilde{\Gamma}) \sin \theta_{i}+(1+\tilde{\Gamma}) \sin \theta_{s}$ and $b=(1+\tilde{\Gamma}) \cos \theta_{s}$ $-(1-\tilde{\Gamma}) \cos \theta_{i}$.

The Fresnel reflection coefficient, $\tilde{\Gamma}$, for a horizontally polarized wave reflecting from a sample material is

$$
\tilde{\Gamma}_{a s}^{-}=\frac{\tilde{n}_{a} \cos \theta_{i}-\tilde{n}_{s} \cos \theta_{t}}{\tilde{n}_{a} \cos \theta_{i}+\tilde{n}_{s} \cos \theta_{t}} .
$$

The indices of refraction for the sample material and atmosphere are given by $\tilde{n}_{s}$ and $\tilde{n}_{a}$, respectively, and $\theta_{t}$ is the transmitted angle relative to the surface normal. The reflection coefficient for vertical polarization, $\tilde{\Gamma}^{+}$, is similar. ${ }^{15}$ Horizontal polarization will be assumed throughout the remainder of this paper.

Although the integral in Eq. (2) is generally intractable, it can be solved numerically for a computer-generated rough surface. Realizations of Gaussian random rough surfaces can be generated $^{27}$ with a given rms height and correlation length, $l_{c}$.

The scattered field depends on the total length of the illuminated surface, $2 L$, which typically spans many wavelengths. Simulations in this paper will use $2 L=100 \lambda_{\max }$, where $\lambda_{\max }$ is the maximum wavelength, corresponding to the lowest frequency in the $\mathrm{THz}$ simulation data.

It should be noted that since this is a scattering model for a one-dimensional surface in a two-dimensional geometry, the scattered power will be different from what would be measured from two-dimensional surface in a three-dimensional space. However, the behavior of the derivative of the scattered power will be similar. ${ }^{10}$

\section{Modeling random rough surfaces}

In order to explore rough surface scattering at $\mathrm{THz}$ wavelengths, surfaces which have random roughness on the order of hundreds of microns are required. Sandpaper was chosen as a representative surface for experimenting with rough surface scattering in the $\mathrm{THz}$ spectrum, due to its commercially availability in a variety grain sizes. Thus, sandpaper provides a convenient control surface for scientific study 


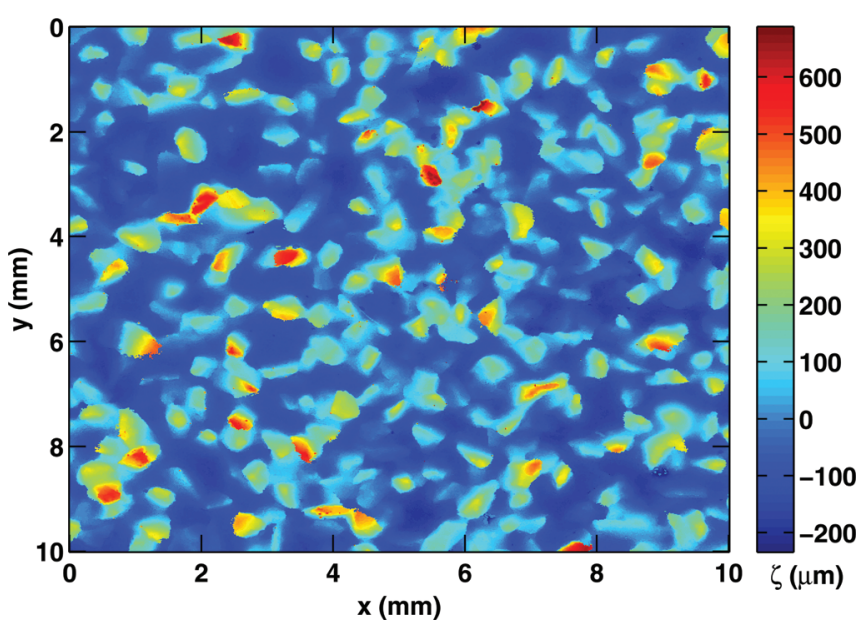

FIG. 2. (Color online) Rough surface height of 40 grit sandpaper (Ref. 28). Surface statistics are listed in Table I.

of random rough surface scattering at $\mathrm{THz}$ wavelengths. The relative roughness of sandpaper is indicated by the grit size, which is inversely proportional to the grain size.

Two sandpaper samples manufactured by Norton will be considered; a rough (40 grit) sandpaper and a much smoother (120 grit) sandpaper. The surface height for each sample was measured by Nanovea, Inc. ${ }^{28}$ The surface height for the 40 and 120 grit samples are shown in Figs. 2 and 3, respectively. The surface height data was used to compute the rms height and correlation length, and results are listed in Table I. The Fraunhofer Criterion in Eq. (1) indicates that for an incident angle of 25 degrees, 40 and 120 grit sandpapers will cause rough surface scattering above $80 \mathrm{GHz}$ and $260 \mathrm{GHz}$, respectively. Therefore, these surfaces will have suitable roughness to demonstrate scattering of $\mathrm{THz}$ waves.

\section{SURFACE SCATTERING MEASUREMENTS}

This section describes how an explosive simulant was prepared and impressed with a rough surface, and how diffuse scattered fields were measured with a THz-TDS

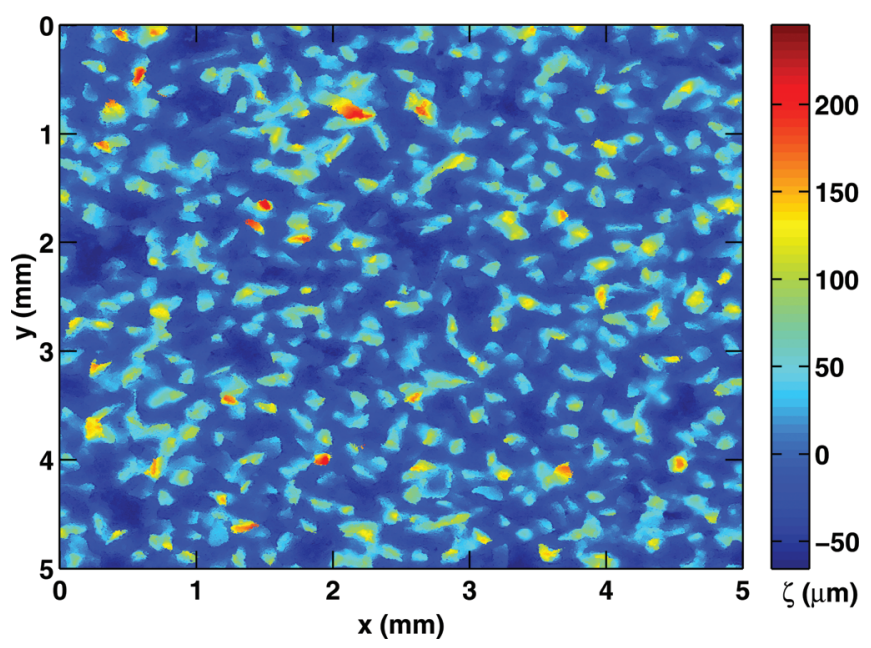

FIG. 3. (Color online) Rough surface height of 120 grit sandpaper (Ref. 28). Surface statistics are listed in Table I. system. Section V will compare measurement data with the simulated scattered field data described earlier.

\section{A. Sample preparation}

The Fraunhofer Criterion and sandpaper roughness statistics indicate that sandpaper can be a useful control surface for studying the effects of rough surface scattering at THz wavelengths. However, the material properties of the various types of sand and glue used to make each of the sandpapers have yet to be characterized at $\mathrm{THz}$ frequencies and are not of interest here. Therefore, sandpaper was used to impress known roughness into a material with known spectral features.

Lactose is known to have strong spectral features at $\mathrm{THz}$ frequencies and is easier to obtain and safer to work with in laboratory experiments than explosives. ${ }^{8,29,30}$ Pellets were made by mixing $\alpha$-monohydrate lactose powder with polyethylene (PE) powder with a 70\%/30\% weight/weight ratio. The mixture was then pressed into pellets using a Specac hydraulic press with a $40 \mathrm{~mm}$ die. A round piece of 40 grit sandpaper was placed inside the die to impose a rough surface on the pellet. The mixture was then pressed with 1 ton for 1 minute, relaxed for 1 minute and then pressed again with 1 ton for 1 minute. Two such pellets with 40 grit roughness were made. A photo of one of the lactose pellets with 40 grit roughness is shown in Fig. 4. The material properties of the lactose/PE mixture were extracted from transmission measurements of thin smooth samples and the extinction coefficient, $\kappa$, is shown in Fig. 5.

\section{B. THz measurement system}

Terahertz scattering measurements were performed using the T-Ray $4000 \mathrm{THz}-\mathrm{TDS}$ system in the Northwest Electromagnetics and Acoustics Research Laboratory (NEAR-Lab) at Portland State University (PSU). The T-Ray 4000 is a commercially developed system manufactured by Picometrix Inc. A fempto-second laser, beam splitter and mirrors are all fully enclosed inside the main module, and fiberoptic and power cables connect the T-Ray 4000 to transmit (Tx) and receive $(\mathrm{Rx})$ heads which are mounted securely on an optical lab table. The laser pulses are guided by the fiberoptic cables and directed to both the Tx and Rx head. Inside the Tx head, the laser pulse interacts with a photoconductive antenna (PCA) to generate a $\mathrm{THz}$ pulse. The $\mathrm{THz}$ pulse is transmitted to the sample and is detected coherently using another PCA inside the Rx head. Figure 6 shows the system configured in a bistatic reflection geometry to measure $\mathrm{THz}$ scattering from a rough surface.

The location of the Tx head and sample holder remained fixed in place while the $\mathrm{Rx}$ head was rotated by a computer-controlled arm to scan over diffuse scattering

TABLE I. Sandpaper rough surface statistics calculated from surface measurements in Figs. 2 and 3 (Ref. 28).

\begin{tabular}{lcc}
\hline \hline Grit & $h(\mu m)$ & $l_{c}(\mu m)$ \\
\hline 40 & 135.2 & 420.0 \\
120 & 40.34 & 156.0 \\
\hline \hline
\end{tabular}




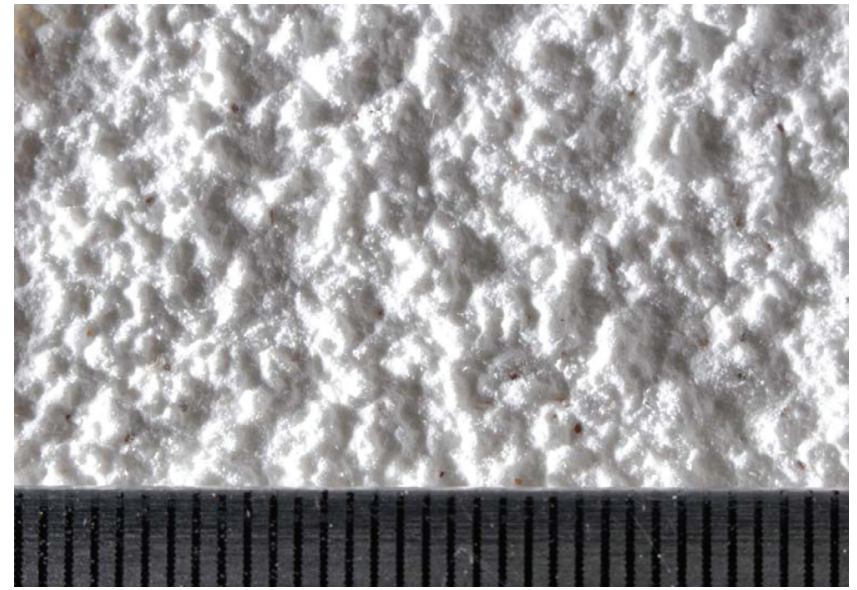

FIG. 4. (Color online) Photo of a lactose pellet impressed with a 40 grit rough surface. The width of the sample in the photo is $3.4 \mathrm{~cm}$, (divisions in $\mathrm{mm})$.

angles. Although not shown in Fig. 6, all of the components were enclosed inside a chamber which was purged with dry nitrogen to remove the effects of water vapor absorption in the air. At each of the scattered angles, the receiver arm paused to average $1000 \mathrm{THz}$ pulse measurements, reducing the noise floor in the measured $\mathrm{THz}$ waveform. The sample stage holding the rough lactose sample was then rotated by computer-controlled motor, to provide a new surface realization and the measurement was repeated. Each measurement was normalized (deconvolved) by a specular reflection measurement from a reference mirror as discussed in Sec. II.

\section{SIGNAL PROCESSING}

The previous two sections described how scattering from dielectric materials with random rough surfaces could be simulated using the KA model and measured using a THz-TDS system. For a fixed incident angle, $\theta_{i}$, each method provides the scattered field as a function of frequency, $f$, and

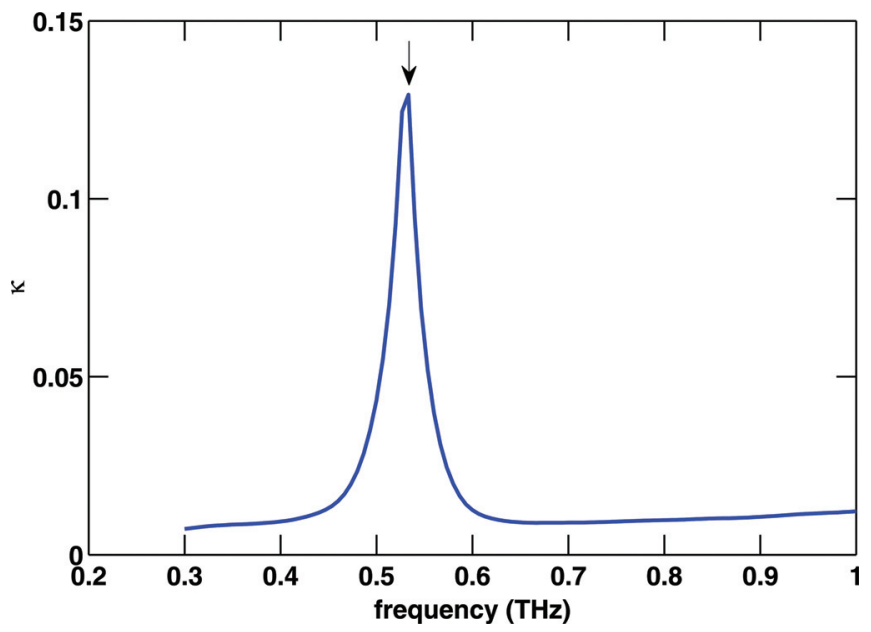

FIG. 5. (Color online) Extinction coefficient, $\kappa$, for $70 \% / 30 \%$ lactose/PE mixture, extracted from transmission measurements shows an absorption peak near $0.54 \mathrm{THz}$

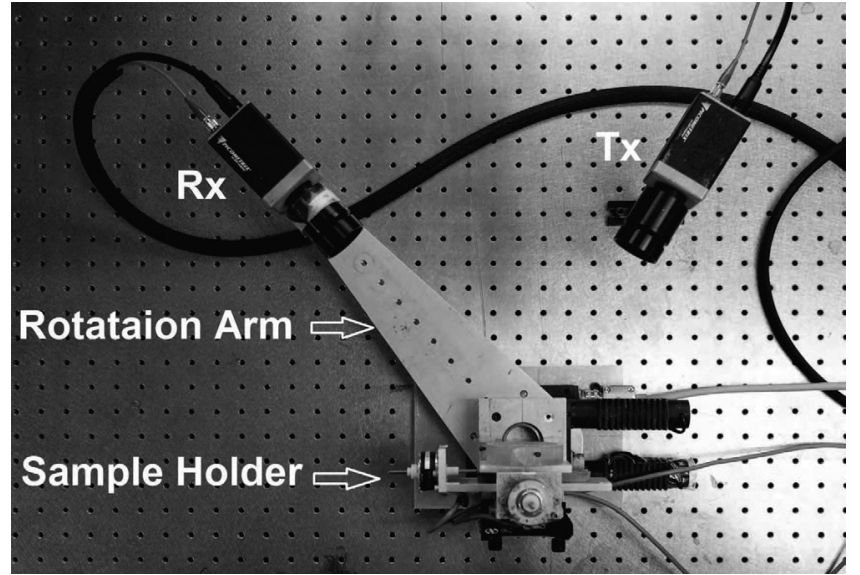

FIG. 6. The Picometrix T-Ray $4000 \mathrm{THz}$ system at the PSU NEAR-Lab. The Tx and Rx heads are shown configured in a bistatic reflection geometry to measure scattering from a rough lactose pellet (Fig. 4) mounted on a sample holder which could be moved to illuminate different areas on the rough surface. The measurement system was enclosed in a chamber (not shown) purged with dry nitrogen.

scattered angle, $\theta_{s}$, for each illuminated surface, $s$. Thus, a set of Monte Carlo simulations or measurements provides the scattered field, $\tilde{E}^{s}\left(f, \theta_{s}, s\right)$. To test the consistency of the results, each process can be repeated many times to form "sets" of surface measurements or Monte Carlo simulations. This section describes how the scattered field data can be processed to extract material features from each data set.

As discussed in Sec. II, for many ERC's the derivative of the reflected power with respect to frequency can reveal spectral features which are useful for material identification. These features appear as peaks in the negative derivative, $D$, of the scattered power as given by

$$
D\left(f, \theta_{s}, s\right)=-\frac{d}{d f} P^{s}\left(f, \theta_{s}, s\right),
$$

where, $P^{s}$, is the scattered power,

$$
P^{s}\left(f, \theta_{s}, s\right)=\tilde{E}^{s}\left(f, \theta_{s}, s\right) \cdot \tilde{E}^{s *}\left(f, \theta_{s}, s\right) .
$$

The scattered field, $\tilde{E}^{s}\left(f, \theta_{s}, s\right)$, is dimensionless because it has been normalized for both the measured and simulated data. Therefore, the scattered power is also dimensionless and the derivative, $D$, has units of time.

The scattered power will contain a slope with respect to frequency due to increasing surface roughness relative to wavelength. This frequency-dependent slope can be removed by detrending the derivative with a third-order polynomial fit.

Averaging over the diffuse scattering angles and surface samples can then reduce the impact of noise introduced by the surface roughness, ${ }^{10}$

$$
D_{a v g}(f)=\frac{1}{N_{s} N_{a}} \sum_{m=1}^{N_{s}} \sum_{n=1}^{N_{a}} D\left(f, \theta_{s, n}, s_{m}\right),
$$

where $N_{a}$ is the number of scattered (observation) angles and $N_{s}$ is the number of surfaces in a set of measurements or 
simulations. Thus, the incoherent noise introduced by the random rough surface can be reduced, while the coherent effect of the material properties becomes more evident.

In addition to averaging, a cepstrum filter may be used to further separate the spectral peaks of the material from the random rough surface noise. ${ }^{10}$ Here, the cepstrum, $\tilde{C}$, will be defined as the Fourier Transform of the average derivative, $D_{\text {avg }}$, from Eq. (6),

$$
\tilde{C}(q)=\mathcal{F}\left\{D_{\text {avg }}(f)\right\},
$$

where $q$ is the quefrency with units of time. Filtering is accomplished by multiplying the cepstrum, $\tilde{C}$, by a (dimensionless) Gaussian filter. The real part of the inverse Fourier transform then gives the filtered derivative,

$$
D_{f}(f)=\mathcal{F}^{-1}\{\tilde{C}(q) \cdot G(q)\},
$$

where $G$ is the zero-mean Gaussian filter,

$$
G(q)=\exp \left(\frac{-q^{2}}{2 \sigma^{2}}\right)
$$

and $\sigma$ is the standard deviation. Since $\tilde{C}$ in Eq. (7) spans positive and negative quefrencies, the standard deviation can be used to define the cutoff quefrency for the low-pass Gaussian filter. Before the Fourier Transform in Eq. (7), the average derivative, $D_{\text {avg }}$, was multiplied by a Hamming window. Likewise, the result of the inverse Fourier Transform in Eq. (8) was divided by the Hamming window.

It is possible that a security screening system need only detect a specific material (or group of materials) that poses a threat. If the characteristic spectral peaks in the extinction coefficient of the target material(s) are wide (low quefrency) relative to the peaks in the surface scattering noise, then a cutoff quefrency may be chosen to remove much of the noise due to scattering and enhance the spectral features. Thus, comparing the cepstrum of the extinction coefficient directly with the cepstrum of the derivative of the scattered power from random rough surfaces (with no spectral features) provides a means of selecting a useful cutoff quefrency. In the following section, the cepstra of several realizations of conductive surfaces are used to assess the distribution of scattering noise from surfaces in the quefrency domain.

\section{RESULTS AND DISCUSSION}

In this section, measured and simulated data are presented to demonstrate the ability to recover spectral features using diffuse scattered power. The KA model is used to simulate scattering from lactose with large-scale (40 grit) surface roughness for a number of surface realizations, and the simulations are validated with a similar set of laboratory measurements from rough lactose surfaces. Simulations are also presented for $\mathrm{C} 4$ explosive material with small-scale (120 grit) roughness. For each case, the ability of cepstrum filtering to enhance the spectral features is demonstrated. All of the simulations and measurements in this section were configured as shown in Fig. 1.

\section{A. Feature extraction from lactose}

This section will demonstrate how averaging and cepstrum filtering can enhance the spectral feature near $0.54 \mathrm{THz}$ in lactose (Fig. 5) impressed with 40 grit roughness as shown in Fig. 4. The incident angle is $\theta_{i}=25^{\circ}$, and the scattered angles range from $\theta_{s}=-12^{\circ}$ to $+12^{\circ}$ in increments of $\Delta \theta_{s}=2^{\circ}$ as depicted in Fig. 1. The total length of each lactose surface is $2 \mathrm{~L}=20.4 \mathrm{~cm}$. In each case, the derivative of the scattered power is averaged for all 13 diffuse observation angles.

The KA model was used to simulate scattering from a lactose surface with the roughness parameters of 40 grit sandpaper shown in Table I. A total of 900 surface realizations were simulated, and the negative derivative of the scattered power with respect to frequency was calculated for 30 sets of 30 surfaces. The results without cepstrum filtering are shown in Fig. 7. The small peak at $0.54 \mathrm{THz}$ which consistently appears in each data set (yellow vertical line) is due to averaging over multiple surfaces and diffuse angles. However, within each data set, the small feature at $0.54 \mathrm{THz}$ is obscured by higher peaks which are due to random surface scattering. Since these peaks are generally narrower than the lactose feature, their amplitude may be reduced with a cepstrum filter.

As discussed earlier, a low-pass cepstrum filter can be designed to reduce the noise by evaluating the normalized magnitude of the cepstrum for the lactose extinction coefficient as shown in Fig. 8 (red dashed line). The KA model was used to simulate 900 conductive surfaces with 40 grit roughness, so that the spectral features due to surface roughness could be isolated from any spectroscopic material features. The magnitude of the cepstrum for 30 sets of 30 conductive surfaces with 40 grit roughness is shown in Fig. 9. The smoothed average of all 30 sets of conductive surfaces is plotted in Fig. 8 (blue solid line) for comparison with the cepstrum of the lactose extinction coefficient. Note that most of the quefrency content of the lactose extinction

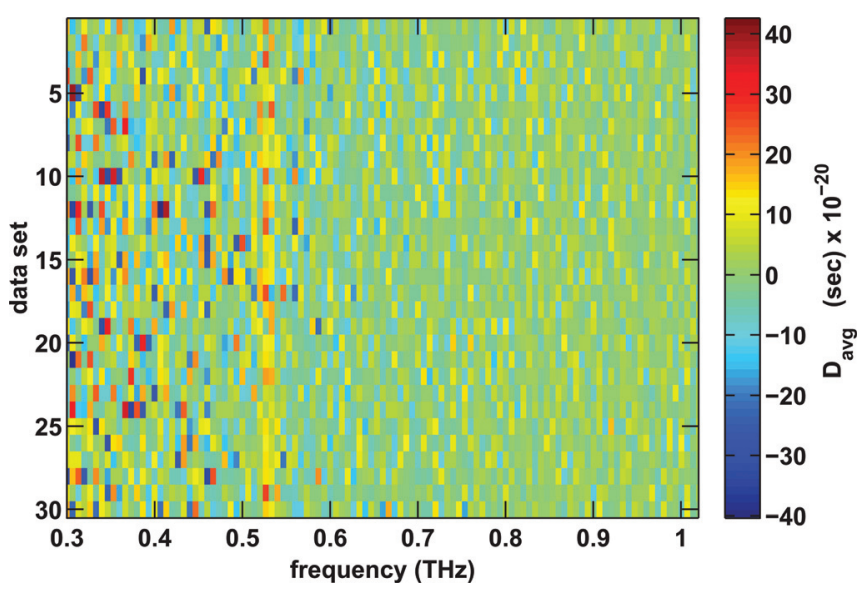

FIG. 7. (Color online) Simulations of the derivative of scattered power from lactose with 40 grit roughness, averaged over 30 surfaces and 13 observation angles with no cepstrum filter. The spectral feature at $0.54 \mathrm{THz}$ is visible in most of the sets (yellow vertical line) but it is obscured by noise due to scattering from the random rough surface. Figure 10 shows the improvement gained by filtering. 


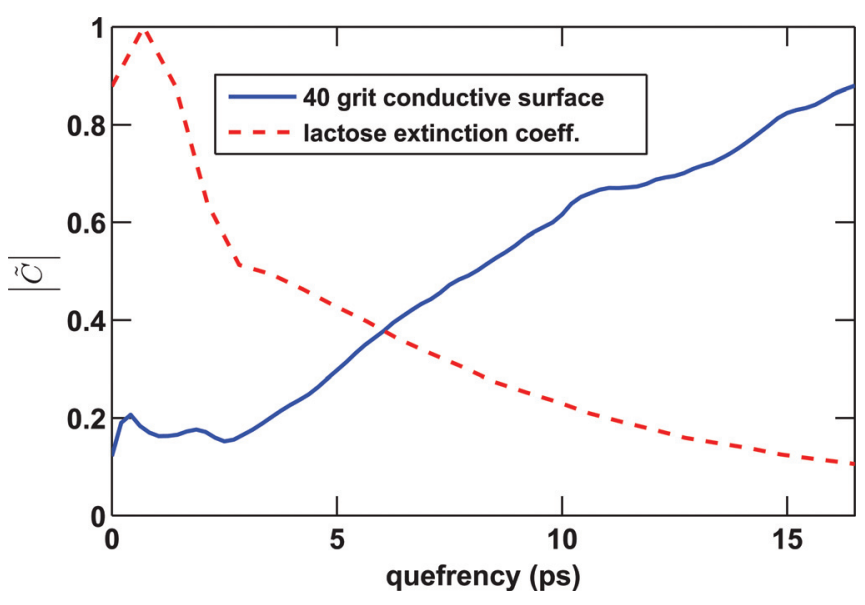

FIG. 8. (Color online) The normalized magnitude of the cepstrum from the lactose extinction coefficient (red dashed line) shows that most of the quefrency content is below $15 \mathrm{ps}$. The normalized average (blue solid line) of all 30 sets of conductive surfaces (from Fig. 9) suggests that a low pass filter may help reduce the random surface scattering noise.

coefficient is below 15 ps while a majority of the quefrency content of the surface roughness is at higher quefrencies. Applying a Gaussian filter with a standard deviation of $15 \mathrm{ps}$ yields the derivative of the scattered power shown in Fig. 10. The spectral feature at $0.54 \mathrm{THz}$ is clearly visible in all of the 30 sets of 30 surfaces (red vertical line).

The values of the derivative, $\mathrm{D}_{\mathrm{f}}$, are quantitatively lower than those in Fig. 7 due to filter attenuation because the Gaussian filter has significant roll-off within the pass band. While other filters may provide more ideal low-pass filtering, these results demonstrate the qualitative improvement that can be gained with a cepstrum filter.

Based on the simulated results presented in Fig. 10 it is expected that a given set of 30 (filtered) rough surface measurements may enhance the spectral peak near $0.54 \mathrm{THz}$. This was tested by measuring the scattering from 1 set of 30 independent surfaces made of lactose with 40 grit roughness using the $\mathrm{THz}$ measurement system described in Sec. III. Figure 11 shows the normalized derivative of the average scattered power with and without a Gaussian cepstrum filter using a standard deviation of 15 ps. As expected the Gaussian filter

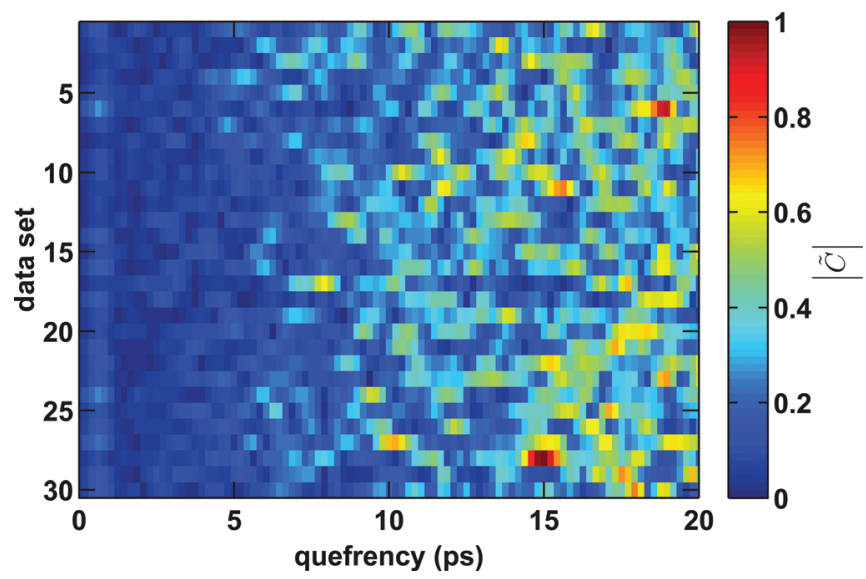

FIG. 9. (Color online) Simulations of the magnitude of the cepstrum for 30 sets of 30 conductive surfaces with 40 grit roughness.

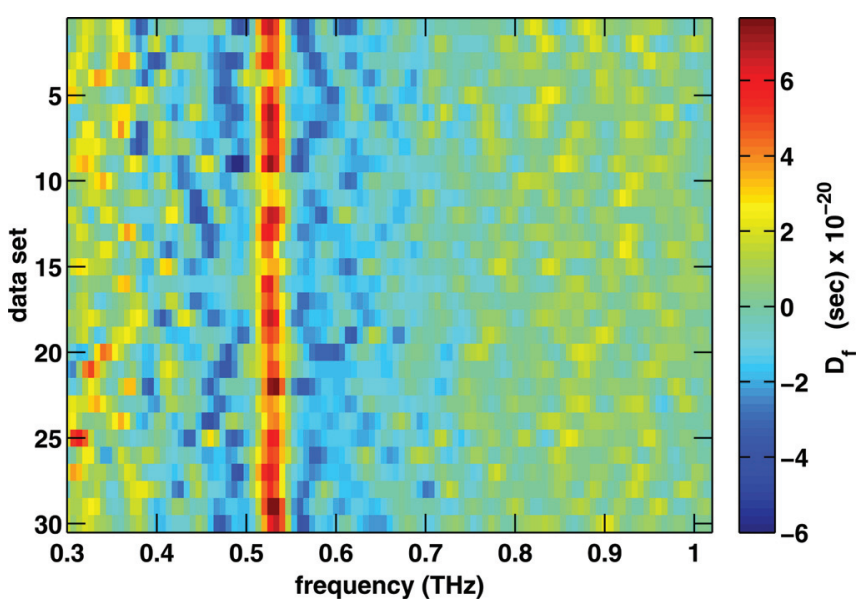

FIG. 10. (Color online) Simulations of the derivative of scattered power from lactose with 40 grit roughness, averaged over 30 surfaces and 13 observation angles. Comparing this result with Fig. 7 shows the surface noise has been reduced and the spectral feature near $0.54 \mathrm{THz}$ is clearly visible.

improves the visibility of the spectral feature at $0.54 \mathrm{THz}$ by attenuating the narrower adjacent peaks which are due to random scattering from the 40 grit random rough surface.

\section{B. Simulated feature extraction from $\mathrm{C} 4$ explosive}

Composition-4 (C4) explosive is of particular interest for security applications due to its accessibility and ease of use by terrorist organizations. In this section we demonstrate the recovery of spectral features of $\mathrm{C} 4$ explosive with smallscale roughness. The effect of averaging over diffuse angles and independent illuminated surfaces is also explored.

The extinction coefficient of $\mathrm{C} 4$ was calculated from the Lorentz parameters in the literature ${ }^{4}$ and is plotted in Fig. 12. There are six absorption peaks located near $0.8,1.1,1.3,1.5$, 2.0, and $2.2 \mathrm{THz}$. The derivative of the reflection coefficient magnitude with respect to frequency can reveal peaks at the same frequencies as the peaks in the extinction coefficient. ${ }^{10}$

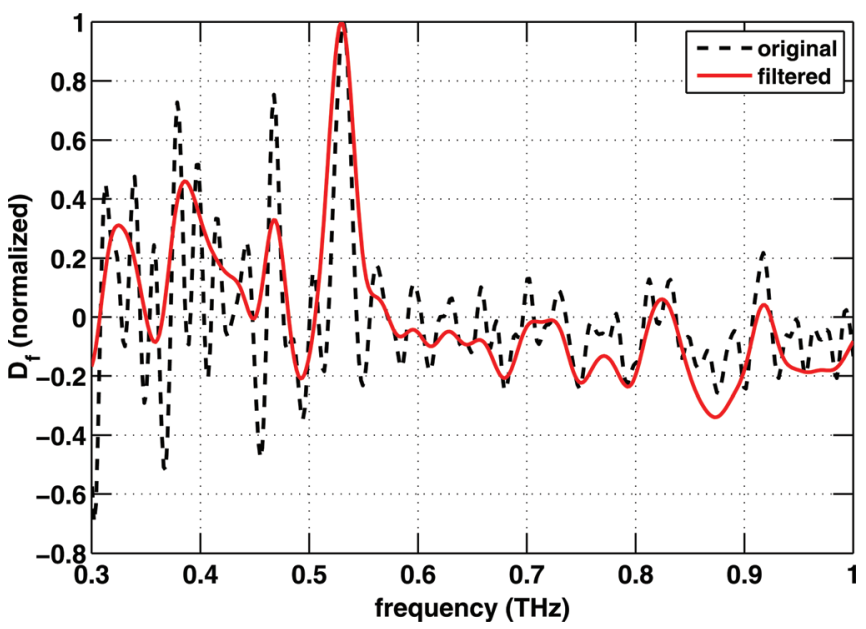

FIG. 11. (Color online) Measurement results for the normalized derivative of scattered power from lactose with 40 grit roughness for one set of 30 surfaces. The spectral feature near $0.54 \mathrm{THz}$ is clearly visible above the noise after filtering (red solid line). The dashed black line shows the result without cepstrum filtering. 


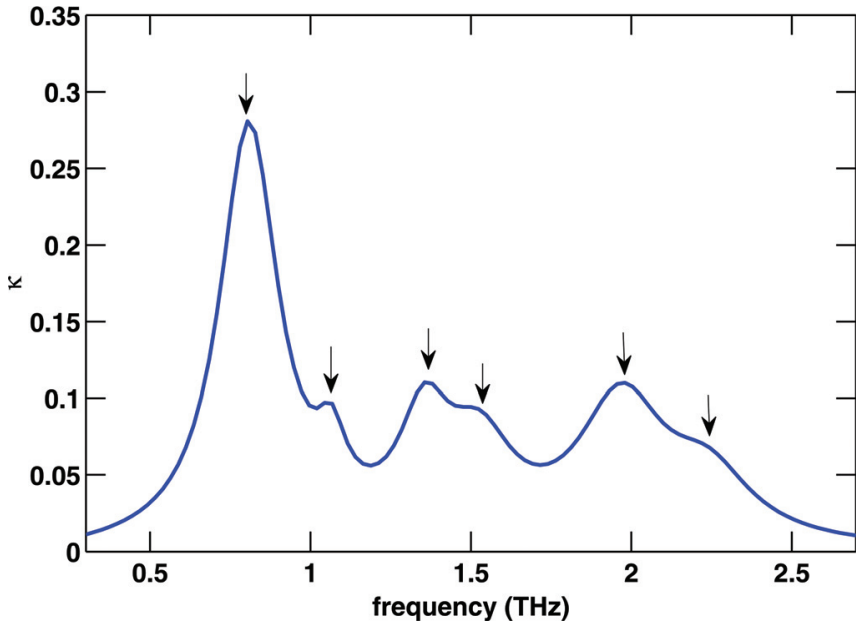

FIG. 12. (Color online) Extinction coefficient, $\kappa$, for C4 Explosive. There are six absorption peaks located near $0.8,1.1,1.3,1.5,2.0$ and $2.2 \mathrm{THz}$.

The KA model was used to simulate scattering from $\mathrm{C} 4$ with the surface roughness statistics of 120 grit sandpaper shown in Table I. The length of each surface was $2 \mathrm{~L}=10 \mathrm{~cm}$ and the incident and scattered angles were the same as those in the previous example. A total of 1500 surfaces were simulated so that a wider variety of averaging options could be explored. Figure 13 shows the results for 30 sets of 15 surfaces measured at only one scattering angle (normal), without a cepstrum filter. No spectral features are visible.

As in the previous example, the cepstra of the extinction coefficient (shown in Fig. 14) and the smoothed average from 15 sets of conductive surfaces with identical roughness (shown in Fig. 15) were used to choose an appropriate cutoff quefrency. Notice that the cepstrum of the $\mathrm{C} 4$ extinction coefficient drops nearly to zero above $5 \mathrm{ps,}$, while the cepstrum of the surface scattering continues to rise at higher quefrencies. Figure 16 shows the result of applying a cepstrum filter with standard deviation of 5 ps to each set of simulations. In most of the data sets the strong spectral feature near $0.8 \mathrm{THz}$ can be observed. As in the previous lactose

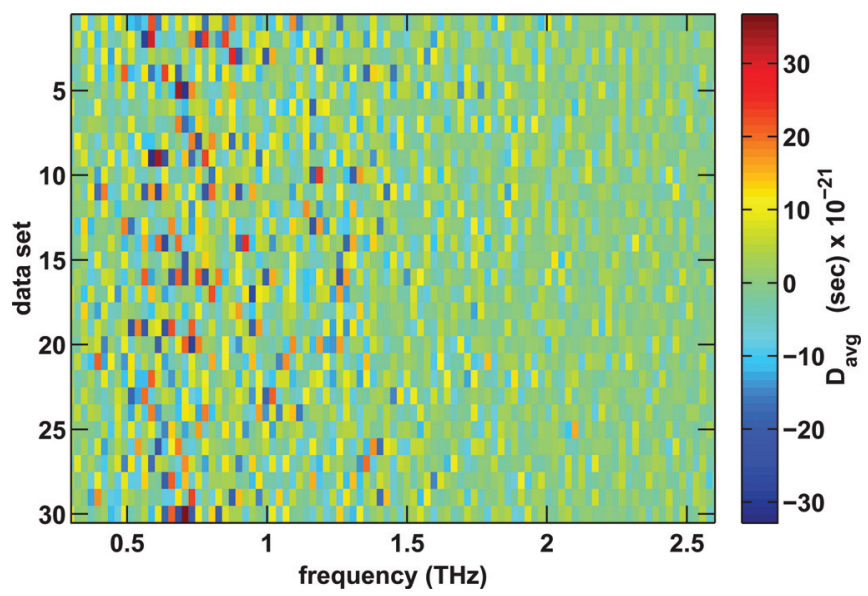

FIG. 13. (Color online) Simulations of the derivative of scattered power at a single observation angle (normal) from C4 explosive with 120 grit roughness, averaged over 15 surfaces with no cepstrum filter. No spectral features are visible. Figure 16 shows the improvement gained by filtering.

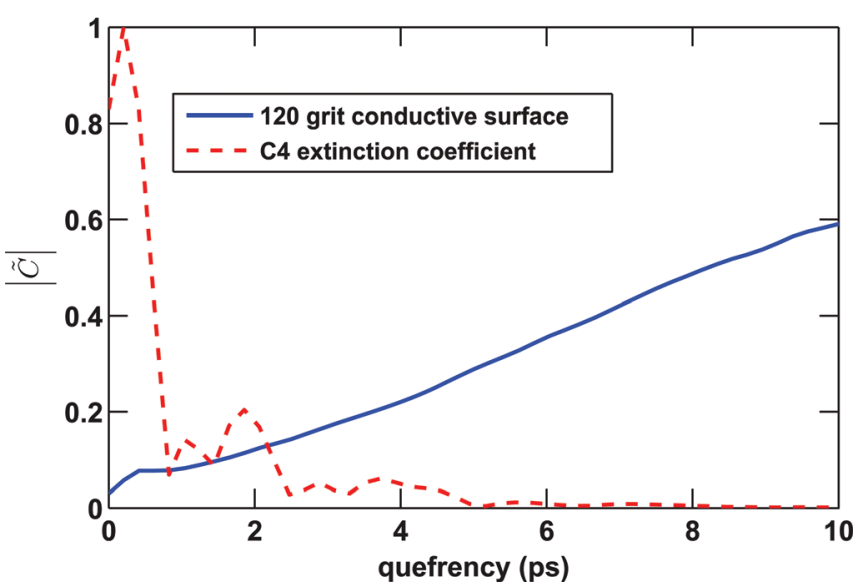

FIG. 14. (Color online) The normalized magnitude of the cepstrum from the $\mathrm{C} 4$ extinction (red dashed line) coefficient shows that most of the quefrency content is below $5 \mathrm{ps}$. The normalized average (blue solid line) of all 15 sets of conductive surfaces (from Fig. 15) suggests that a low pass filter may help reduce the random surface scattering noise.

example, the values of the derivative, $\mathrm{D}_{\mathrm{f}}$, are quantitatively lower than those in Fig. 13 due to filter attenuation.

Figure 17 shows the improvement gained by averaging overall of the scattering angles between $-12^{\circ}$ and $12^{\circ}$. The feature at $0.8 \mathrm{THz}$ becomes more pronounced (red vertical line) and some minor features (near 1.3 and $2.0 \mathrm{THz}$ ) become more apparent (green vertical lines).

Figure 18 shows that further improvements can be gained by including more surfaces in each data set. When averaging is done with 50 surfaces the smaller features near $1.1,1.5$, and 2.2 are consistently more clear in each data set (green vertical lines).

\section{Discussion}

The measurements and simulation data presented in this section demonstrated that $\mathrm{THz}$ spectral features may be consistently recovered using diffuse reflections from rough surface scattering. Application of a low-pass cepstrum filter improves detection of spectral peaks that are wide relative to noise peaks due to rough surface scattering. Increasing the number of diffuse angles and independent illuminated surfaces also improves the ability to identify material features.

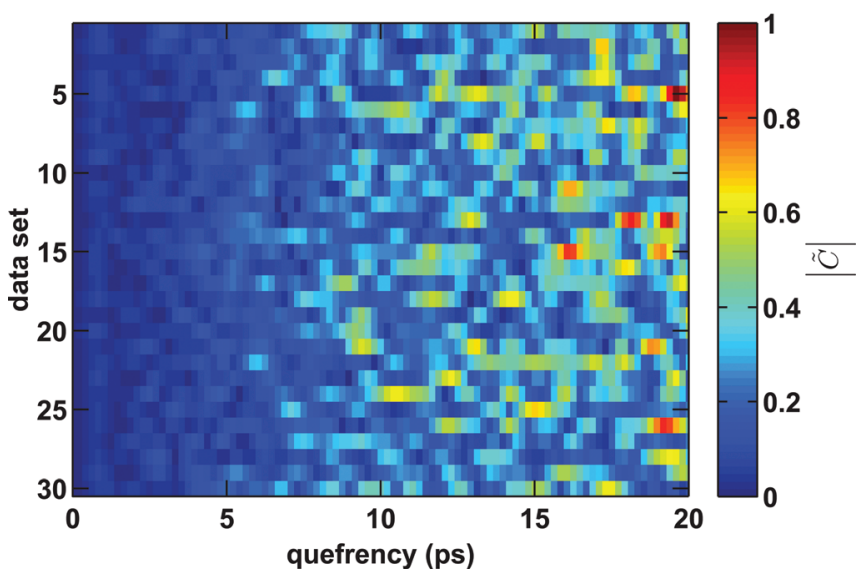

FIG. 15. (Color online) Simulations of the magnitude of the cepstrum for 30 sets of 15 conductive surfaces with 120 grit roughness. 


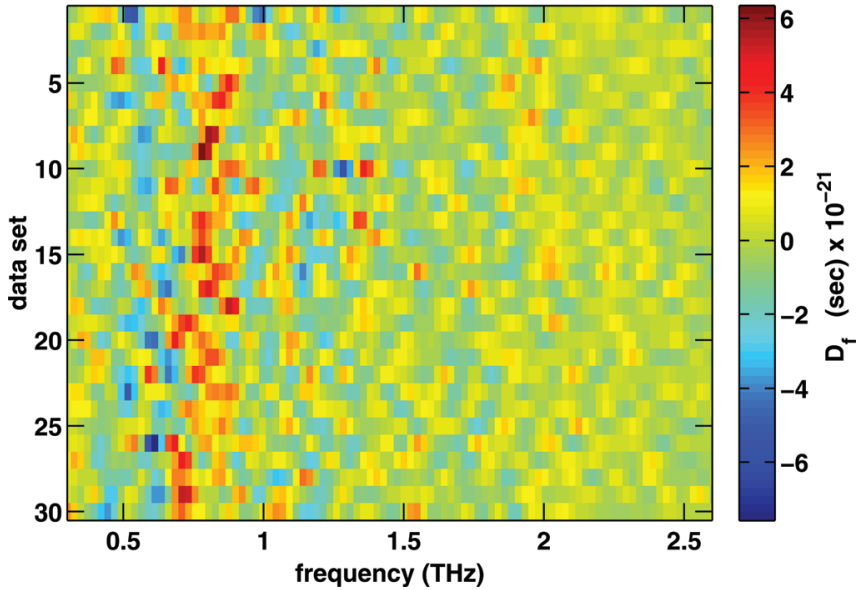

FIG. 16. (Color online) Simulations of the derivative of scattered power from $\mathrm{C} 4$ with 120 grit roughness, averaged over 15 surfaces at one observation angle (normal) with a low-pass cepstrum filter below 5 ps. Comparison with Fig. 13 shows that the spectral peak at $0.8 \mathrm{THz}$ is more visible after filtering.

In these examples a Gaussian function was used to filter the cepstrum, and the standard deviation served as the cutoff quefrency. The slow roll-off of the Gaussian caused attenuation of the derivative values at all quefrencies - including those within the pass band. These results demonstrate that even a relatively poor (non-ideal) filter can provide significant benefits. Future efforts could explore other filters.

It should be noted that the number of measurements needed in real three-dimensional (3D) measurement systems should be less than what is indicated by $2 \mathrm{D}$ simulations (such as were used here) by a factor proportional to the rough surface correlation length, $l_{c} \cdot{ }^{31,32}$ For example, a square illuminated patch of surface with dimensions $10 l_{c} \times 10 l_{c}$ would require $10 \times$ fewer surfaces than would be required by a $2 \mathrm{D}$ simulation of scattering from a $1 \mathrm{D}$ strip of length $10 l_{c}$. The area of a surface illuminated by a THz-TDS transmit antenna will not be rectangular and will change with the frequency, but the scaling difference between 3D measurements and 2D

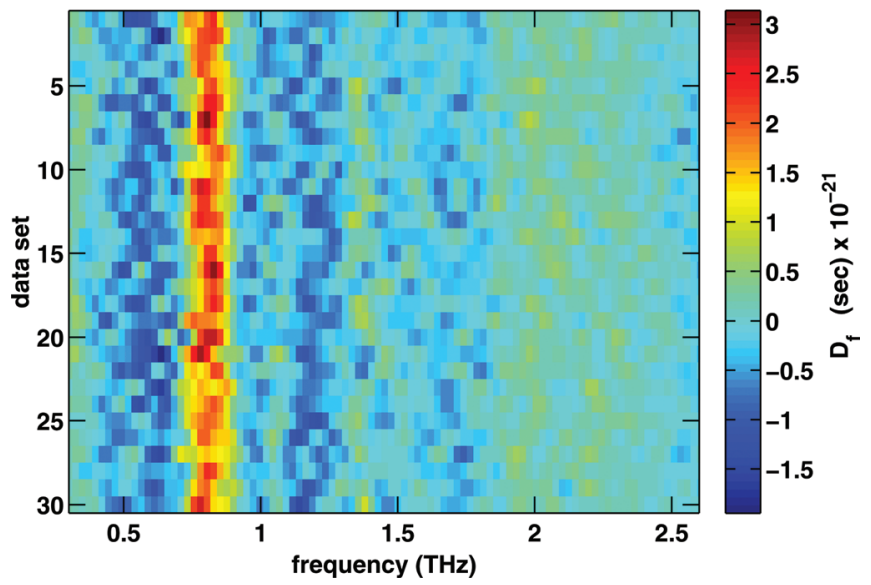

FIG. 17. (Color online) Simulations of the derivative of scattered power from $\mathrm{C} 4$ with 120 grit roughness, averaged over 15 surfaces and 13 observation angles (between -12 degrees and $12^{\circ}$ in $2^{\circ}$ increments) with a low-pass cepstrum filter below 5 ps. Comparison with Fig. 16 shows that in addition to the peak at $0.8 \mathrm{THz}$ the spectral peaks near 1.3 and $2.0 \mathrm{THz}$ is more visible (green vertical lines).

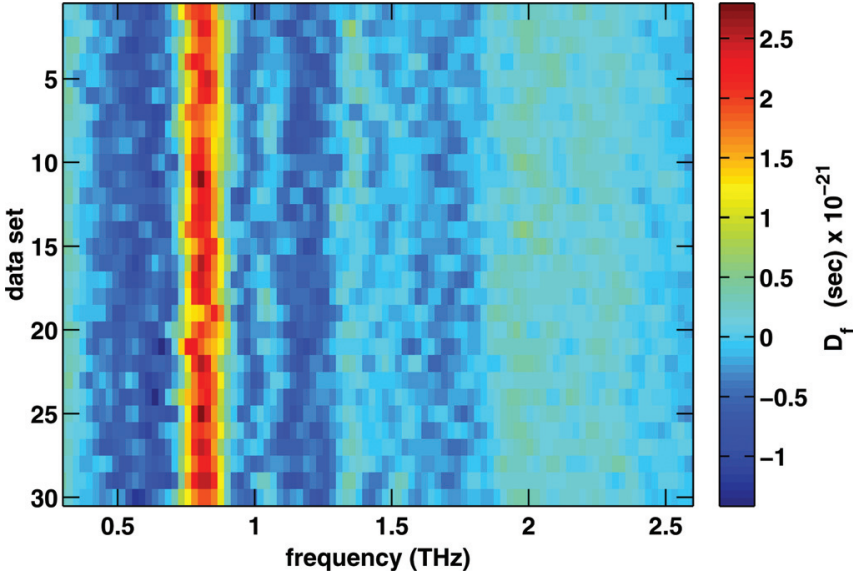

FIG. 18. (Color online) Simulations of the derivative of scattered power from $\mathrm{C} 4$ with 120 grit roughness, averaged over 50 surfaces and 13 observation angles (between $-12^{\circ}$ and $12^{\circ}$ in $2^{\circ}$ increments) with a low-pass cepstrum filter below 5 ps. Comparison with Fig. 16 shows that including more surfaces in each data set makes all of the features more clear and consistent.

simulations indicates that the number of surface samples required for material detection will be less than what is indicated by the simulations.

\section{CONCLUSION}

In the past decade $\mathrm{THz}$ spectroscopy has emerged as a promising new technology with applications including detection of explosives and illicit drugs. However, the opacity of most materials of interest at these frequencies is likely to limit practical systems to reflection geometry. In addition, the roughness of most materials relative to $\mathrm{THz}$ wavelengths motivates us to consider ways to make use of diffuse scattering. Our earlier work ${ }^{10}$ demonstrated that spectral features could be extracted from diffuse scattering averaged over a number of observation angles and illuminated areas, and that a cepstrum filter could be used to reduce noise due to rough surface scattering.

This paper expanded on these concepts by using the cepstra of the extinction coefficient of the target material and the rough surface scattering to choose an optimal cutoff quefrency for a cepstrum filter. The Kirchhoff Approximation was used to quickly simulate scattering from many sets of random rough surface realizations at a number of scattering angles and frequencies from rough dielectric materials. The effects of averaging and cepstrum filtering were demonstrated for two materials with different roughnesses. Terahertz scattering measurements from a dielectric impressed with a rough surface also showed the value of the cepstrum filter. The signal processing methods presented here may be useful for the design of future $\mathrm{THz}$ security screening systems.

${ }^{1}$ D. L. Woolard, E. R. Brown, M. Pepper, and M. Kemp, Proc. IEEE 93, 1722 (2005).

${ }^{2}$ M. Kemp, P. Taday, B. Cole, J. Cluff, A. Fitzgerald, and W. Tribe, Proc. SPIE 5070, 44 (2003).

${ }^{3}$ X.-C. Zhang, Phys. Medicine Biol. 47, 3667 (2002).

${ }^{4}$ K. Yamamoto, M. Yamaguchi, F. Miyamaru, M. Tani, M. Hangyo, T. Ikeda, A. Matsushita, K. Koide, M. Tatsuno, and Y. Minami, Jpn. J. Appl. Phys. Part 2 Lett. 43, 414 (2004). 
${ }^{5}$ L. Borne and B. A., "Effects of crystal internal defects on projectile impact initiation," Tech. Rep. (French-German Research Institute of Saint-Louis (ISL), P.O. Box 34 - F 68301 Saint-Louis Cedex, 2002).

${ }^{6}$ J. E. Reaugh, "Grain-scale dynamics in explosives," Technical Report 30, 2002.

${ }^{7}$ J. E. Reaugh, "Checking out the hotspots," technical report, 2003.

${ }^{8}$ M. H. Arbab, A. Chen, E. I. Thorsos, D. P. Winebrenner, and L. M. Zurk, Proc. SPIE 6893, 68930 (2008).

${ }^{9}$ S. Henry, G. Kniffin, S. Schecklman, L. Zurk, and A. Chen, "Measurement and modeling of rough surface effects on terahertz spectroscopy and imaging," Proceedings of SPIE - The International Society for Optical Engineering, Terahertz Technology and Applications III 7601, 760108-1 (2010).

${ }^{10}$ G. Sundberg, L. M. Zurk, S. Schecklman, and S. Henry, IEEE Trans. Geosci. Remote Sens. 48, 3709 (2010).

${ }^{11}$ A. V. Oppenheim and R. W. Schafer, "From frequency to quefrency: A history of the cepstrum," IEEE Signal Processing Magazine 21, 95-99,106 (2004).

${ }^{12}$ Y. Dikmelik, J. B. Spicer, M. J. Fitch, and R. Osiander, Opt. Lett. 31, 3653 (2006).

${ }^{13}$ Y. Dikmelik, M. J. Fitch, M. R. Leahy-Hoppa, and R. Osiander, Proc. SPIE 6549, 65490 (2007)

${ }^{14}$ M. R. Leahy-Hoppa, M. J. Fitch, and R. Osiander, Proc. SPIE 6893, 689305 (2008).

${ }^{15}$ S. Mickan and X.-C. Zhang, Int. J. High Speed Electron. Syst. 13, 601 (2003).

${ }^{16}$ K. Kawase, Y. Ogawa, Y. Watanabe, and H. Inoue, Opt. Express 11, 2549 (2003).

${ }^{17}$ W. R. Tribe, D. A. Newnham, P. F. Taday, and M. C. Kemp, Proc. SPIE 5354, 168 (2004)
18“"The database," available at www.thzdb.org.

${ }^{19}$ H. Zhong, A. Redo-Sanchez, and X.-C. Zhang, Opt. Express 14, 9130 (2006).

${ }^{20} \mathrm{H}$. Zhong, "Terahertz wave reflective sensing and imaging," Ph.D. thesis, (Rensselaer Polytechnic Institute, 2006).

${ }^{21}$ H. Zhong, A. Redo-Sanchez, and X.-C. Zhang, Int. J. High Speed Electron. Syst. 17, 239 (2007).

${ }^{22} \mathrm{P}$. Beckmann and A. Spizzichino, The Scattering of Electromagnetic Waves from Rough Surfaces, (Artech House, Boston, 1987).

${ }^{23}$ F. T. Ulaby, R. K. Moore, and A. K. Fung, Microwave Remote Sensing: Active and Passive, vol. 2,. (Addison-Wesley Pub., Reading, MA, 1986).

${ }^{24}$ E. Thorsos and D. R. Jackson, J. Acoustic Soc. Am., 83, 78 (1988).

${ }^{25}$ L. Tsang, J. A. Kong, and R. T. Shin, Theory of Microwave Remote Sensing, (Wiley, New York, 1985).

${ }^{26}$ L. Tsang and J. A. Kong, Scattering of Electromagnetic Waves, (Wiley, New York, 2001) Vol. 3.

${ }^{27}$ L. Tsang, J. A. Kong, K.-H. Ding, and C. O. Ao, Scattering of Electromagnetic Waves, Volume 2, Numerical Simulations (John Wiley and Sons, 2001).

${ }^{28}$ Nanovea, 6 Morgan, Suite 156, Irvine, CA 92618, available at www.nanovea.com.

${ }^{29}$ Y. Shen, P. F. Taday, and M. C. Kemp, Proc. SPIE 5619, 82 (2004).

${ }^{30}$ M. Walther, M. R. Freeman, and F. A. Hegmann, Appl. Phys. Lett. 87, 1 (2005).

${ }^{31}$ W. Makinde, N. Favretto-Cristini, and E. de Bazelaire, Geophys. Prospect. 53, 373 (2005).

${ }^{32}$ C. Walter and C. Mitterer, Surface Coatings Technol., 203, 3286 (2009). 